\title{
Induced Orientational Effects in Relaxation of Polymer Melts
}

\author{
J. M. Deutsch and J. H. Pixley \\ Department of Physics, University of California, Santa Cruz, California 95064
}

(Dated: March 11, 2022)

\begin{abstract}
We study stress relaxation in bi-disperse entangled polymer solutions. Shorter chains embedded in a majority of longer ones are known to be oriented by coupling to them. We analyze the mechanism for this both by computer simulation and theoretically. We show that the results can be understood in terms of stress fluctuations in a polymer melt and chain screening. Stress fluctuations are frozen on the relaxation time of the longer chains, and these will induce strong orientational couplings in the shorter chains.
\end{abstract}

PACS numbers:

Polymer molecules in concentrated solution or melts have very unusual visco-elastic properties [1] and are the subject of much research [2, 3]. After shearing such a system of long chains, the modulus has a long plateau that depends strongly on chain length, yet the height of the plateau appears almost independent of it.

The most successful model in explaining the experimental data is the "reptation" model of de Gennes [4]. The initial work concentrated on the case of a polymer in a background network such as a gel which is most easily understood, but since then, reptation has had many applications to polymer solutions. The idea is that topological effects restrict the movement of a polymer to a tube by virtue of entanglements thereby suppressing transverse motion for scales larger than the "tube diameter". However chain ends can move freely forming new tube. A polymer then has a curvilinear diffusion coefficient inversely proportional to its length $L$, so that the time to form a new tube $\propto L^{3}$. This is also expected to be the relaxation time in a polymer melt.

To compare this to experimental data on the viscosity, we recall that the elastic modulus $G(t)$ is related to the viscosity $\eta$ through

$$
\eta \propto \int_{0}^{\infty} G(t) d t
$$

Because the plateau is independent of $L$, this gives a viscosity $\propto L^{3}$. The experimental data in the entangled regime fits better with $L^{3.4}$. The explanation for this discrepancy has yielded a number of theoretical explanations. The two main reasons are both legitimate extensions of de Gennes original idea. First, finite size effects [5, 6], such as tube length fluctuations, yield a larger slope for this quantity. Second, many body effects that give rise to chain screening [7, 8,9$]$ require modification of the original single chain model. Researchers have been able to fit experimental data separately using either idea and it is clear that in fact both are real and experimentally contribute.

That many body effects must be important can be seen by considering what happens if the chains were all independent. Then density fluctuations of the system would become very large and the system would be very compressible. A real system has excluded volume interactions which leads to a small compressibility and chain screening, that is, ideal chain statistics on a large scale 2]. The maintenance of screening leads to an increase in free energy when the chain moves out of a tube [7, 8, 9]. To maintain constant density, this produces a stress field whose strength grows linearly with the new tube. This implies that the motion will be activated as the original tube is vacated. The stress is relieved by other chains moving into or out-of the stressed regions.

This mechanism causes chains to follow the stress trails of other chains because as a chain leaves a tube, it leaves a vacancy which is attractive to other chains. The memory for this stress mechanism will decay with a longer relaxation time than the time for the chain to vacate a tube. This was proposed as an explanation for the difference between diffusion and stress relaxation [10].

The effects of interaction of chains can perhaps most easily be seen by the fact that they induce partial alignment with each other. Many experiments have shown that there is strong induced orientational coupling of smaller chains under stretching of a system containing mainly longer ones, for example in bi-disperse polymer melts 11] where the ratio of the orientation of the smaller chains to the longer host chains was found to be $\epsilon=0.45$ which is quite large. It is the purpose of this work to show that this phenomenon can at least partially be accounted for by the same mechanism responsible for the many-body effects related to chain screening mentioned above. Earlier work looking at this by simulation techniques could reproduce similar orientational responses by means of a direct simulation of these systems [12]. This work should help to clarify the origin of this effect theoretically.

We start by performing computer simulations similar to those done earlier on many body effects for long polymer chains [7, 8]. Consider two chain lengths $L_{1}$ and $L_{2}$. We will assume reptation dynamics [13] for moving chains. Chains live on a cubic lattice in a $B \times B \times B$ box. In each step, the head or tail monomer of a chain is chosen at random, and then an attempt is made to reattach it, in a random orientation, to the other end. It is rejected only if the new lattice position is occupied. We assume a curvilinear diffusion coefficient $\propto 1 / L_{i}, i=1,2$, which is expected from reptation [4]. Translated to a 
Monte-Carlo simulation, we say that the probability of choosing a chain is $\propto 1 / L_{i}$. As discussed above, this system had been analyzed numerically in considerable detail [8] and shows activated motion in agreement with the theoretical predictions [8, 9] giving a relaxation time $\sim \exp \left(\left(L / L^{*}\right)^{2 / 3}\right)$ for very long chains, where $L^{*}$ is a constant that depends on the density of the system. In a real melt it would more directly depend on the plateau modulus of the melt.

For the simulation to be relevant it must be in the same universality class for statics and dynamics as that of a real melt. However the lattice model does not allow monomers to simultaneously rearrange themselves as monomers reptate to new sites. In a real system, such local rearrangement will happen as is required by the incompressibility of the system. A consequence of this is that stress will be created by these rearrangements leading to an increase in free energy that turns out to be local [8]. This local increase in the free energy is precisely what one simulates with reptation dynamics. Therefore we do expect the same essential physical effects in these lattice simulations as well.

To understand memory effects in such systems, we prepare the simulation in a sheared state, where the orientation of each monomer is now anisotropic. We do this by biasing the Monte-Carlo algorithm so that it is more likely to make steps in the z-direction rather than the other two. The system is equilibrated in this state by letting it evolve for many relaxation times. At this point, the effects of shearing are turned off and directions of motion are chosen to be isotropic.

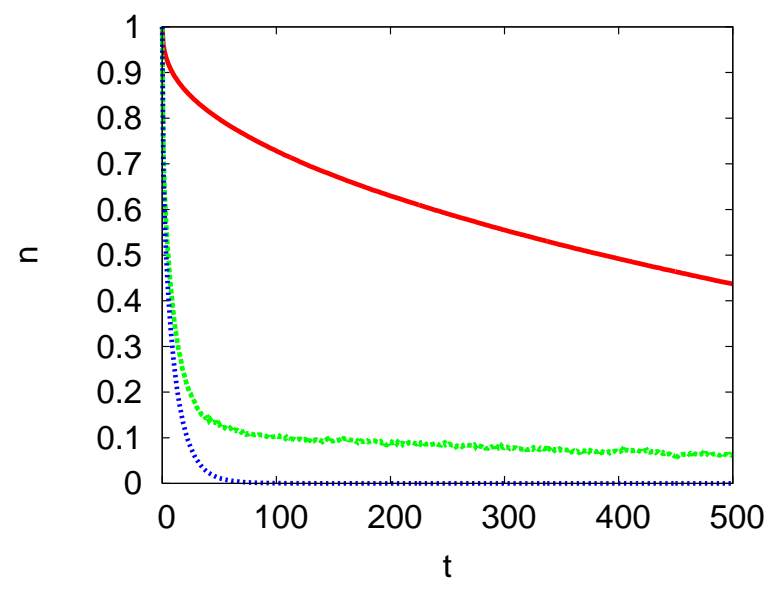

FIG. 1: (Color Online) The decay of the average birefringence for a simulation using reptational motion in a polymer melt with two different chain lengths. The top plot, $L_{2}=256$, and the middle plot $L_{1}=64$. The lower plot is the average amount of $L_{1}$ length chain left in the tube at time $t$.

To illustrate the effect, we shall analyze what happens in a polydisperse system made up predominantly of long chains $L_{2}$ with a minority of shorter chains $L_{1}$. For example, we consider a box of size $B=16$ with 9 chains of length $L_{2}=256$ and one chain of length $L_{1}=64$, with a number density of $\approx 0.58$. We monitor two kinds of quantities. First we monitor $l(t)$, the average amount of the original tube left at time $t$. We also measure the birefringence which is the deviation of polymer links from an isotropic state. We define this as

$$
n \equiv 2 n_{z}-n_{x}-n_{y}
$$

where $n_{x}, n_{y}$, and $n_{z}$ are the fraction of monomers going in the $x, y$, and $z$ directions respectively.

In the Doi Edwards model, the number of entanglement points still remaining after time $t$ is a measure of the stress of the system. Hence in their model the length of original tube $l(t)$ is proportional to the elastic modulus. However if there are collective effects, even after a chain has left its original tube, it can still have a residual orientational bias. Therefore we should monitor more directly, the birefringence of the system, which for small forcing will be linear will the stress of the system.



FIG. 2: (Color Online) The residual birefringence $r(t)$ and the average amount of chain left in a tube $l(t)$ are shown. As is clear, the two curves closely follow each other.

In Fig. 1 we plot the decay of the birefringence separately for the longer chains (upper plot) and the shorter chain (middle plot). The lower plot is $l(t)$, the amount of chain left in the original tube.

In the original reptation picture, one expects that the birefringence should be proportional to the chain left in the tube. Clearly for the shorter chains, this is not the case. The birefringence quickly decays initially, following $l(t)$ quite closely, however it appears to level off at a nonzero value. After this is decays, but at a much longer time scale. We will analyze what mechanism gives rise to such a striking effect.

In the initially biased preparation of the system, links are more likely to be oriented in the $z$ direction, therefore as the polymer chain leaves its tube, it will see a biased environment. In the extreme case of an environment oriented as rods in the $z$ direction, the chain will also be 
biased in the same direction as well [14]. In the case of smaller orientational biases, it is then expected to have an effect which is linear in the average birefringence of the chains. If the surrounding chains are much longer than the shorter one, it will see an almost constant orientational bias as it moves. Even after it has completely left its original tube it will possess a residual orientational bias. This will then slowly decay with time at the same rate as the bias of the longer chains.

Therefore we should modify the non-interacting reptational hypothesis by saying that the orientational bias of the shorter chains is the amount of chain left in the tube at time $t$ plus the bias due to the environment. This is precisely the same assumption made in reptationbased treatments of this effect made previously [15] So we can define the "residual birefringence" due to the shorter chains as

$$
r(t) \equiv n_{1}(t)-\epsilon n_{2}(t)
$$

where $\epsilon$ is the fraction of orientational coupling induced by the longer chains, as has been measured experimentally [11]. After subtracting out the background bias from the longer chains, we are left with the bias from chains that have yet to leave their tube. We therefore expect $r(t) \propto l(t)$. To test if this is the case, we find the value of $\epsilon$ that best eliminates the longer time decay, and then plot $r(t)$ and $l(t)$ as shown in Fig. 2, It is clear that the two curves fit each other quite well. In this case $\epsilon=0.14$.

Therefore we have gained a good understanding of how orientation decays in this polydisperse polymer lattice simulation. Shorter chains continue to have substantial anisotropy even after completely leaving their original environments by the influence of the surrounding longer chains, that still have not relaxed to an unbiased state.

This is in accord with earlier experimental and theoretical work on these systems. This orientational bias is an effect identical to what is seen in more microscopic accurate simulation on effectively shorter chains 12. The degree of orientational coupling is given by the factor $\epsilon$, which given the density of this system, appears to be consistent with the value of coupling found in the more microscopic approach [12].

Now we turn to the relation between this lattice simulation and real polymer melts. There are two idealizations of the simulation that need to be addressed that stem from a lack of continuous translational symmetry for lattice models. The initial conditions from which the system relaxes, and the inability of surrounding chains to simultaneously rearrange themselves so as to accommodate new chain.

First, in a real experiment, the elastic modulus is defined as the stress relaxation observed after a small and sudden deformation of the material. After such a deformation, the system is in a nonequilibrium state, and the polymer chains will then move to restore themselves to equilibrium statistics. The simulation here is started in a deformed state and is then relaxed. The deformed state has been obtained by equilibrating the chains so that their directions are biased in the $z$ direction. This is not identical to a real experiment because lattice chains cannot be continuously deformed due to a lack of continuous translational symmetry. A test of the validity of such a procedure is to ask if we are in the small deformation, linear regime. We therefore test to determine if the relaxation of the system is linear in the initial birefringence. We calculated the integral of $n(t)$, normalized by its initial value, for two different initial biases, and found that indeed this was the case. For example, we considered a melt of chains of length 64 with a 0.58 of the lattice sites occupied. When the bias was reduced by $38 \%$, this integral only changed by $2.3 \%$.

Second, as mentioned above, there are no multimonomer moves with reptation dynamics, and so neighboring chains are unable to rearrange themselves around new tube being formed. This causes an increase in the local chain density of a region. In reality, what happens instead is that the system is almost incompressible, so that stress and strain are created by the introduction of new monomers into a region. These effects in equilibrium have been analyzed in detail previously [8, 9] and give rise to the same behavior as in the simulations.

In the simulation described here, the reason that the polymer chain acquires an anisotropy is due to the influence of the surrounding medium. The surrounding medium is inhomogeneous and the density of the medium is frozen in on the time scale of a relaxation time of the majority (longer) chains. The anisotropy of density fluctuations cause the polymer to orient in the same direction. In a real melt, the analogous quantities to consider are stress fluctuations as was analyzed by Rubinstein and Semenov [9] when the system was isotropic. We will consider the effect of these fluctuations in a stressed polymer melt. The network stress $\sigma(\mathbf{r})$ will be frozen in for timescales less than the relaxation time. When the end of a chain enters a certain region, the network will deform by an amount $\Delta u(\mathbf{r})$. The excess free energy is then

$$
F \sim \int \Delta u(\mathbf{r}) \sigma(\mathbf{r}) d^{3} \mathbf{r} .
$$

The typical energy of a fluctuation of order an entanglement length $\propto \sqrt{N_{e}}$, must obey Boltzmann statistics and is therefore of order $k_{B} T[\underline{9}]$. At the same time, the energy of such a fluctuation in a volume $V \propto N_{e}^{3 / 2}$ is

$$
W \sim \frac{V \sigma^{2}}{K}
$$

where $K$ is the (longitudinal) elastic modulus of the network and is $\propto k_{B} T / N_{e}$ [2].

The increase in free energy in placing additional chain in a new region is responsible for the exponential dependence of relaxation time on chain length as has been previously analyzed [8, 9]. However what we are interested in here is the fluctuation in the additional free energy that must be payed by placing an entanglement length of chain 
in a new region. The average deformation $u \sim N_{e} / V$ and Using Eqs. 4 and [5, this gives [16], $\delta F \sim T / N_{e}^{1 / 4}$.

We can therefore think about the elastic fluctuations as causing a random potential with an R.M.S. $\propto N_{e}^{-1 / 4}$ that the chain interacts with. We now ask what happens when the network is deformed. Suppose an affine deformation $e$ is applied that is of order 1 . The random potential will now also be deformed, just as the density had been deformed in the simulations. So we expect an anisotropy in the potential also of order $k_{B} T N_{e}^{-1 / 4}$. Therefore a chain segment of length $N_{e}$ will no longer explore its surroundings isotropically, but have a bias of order $1 / N_{e}^{1 / 4}$ in the $\mathrm{z}$ direction. Therefore the birefringence of the chains in this new region will, more generally be $\sim e N_{e}^{-1 / 4}$.

To understand this more quantitatively, write the correlation function for the random potential $\phi(\mathbf{r})$ as

$$
\left\langle\phi(\mathbf{r}) \phi\left(\mathbf{r}^{\prime}\right)\right\rangle=v\left(\mathbf{r}-\mathbf{r}^{\prime}\right) .
$$

For a single chain in this environment, the annealed and quenched averages yield the same results [17]. Averaging over realizations of the random potential leads a weak attraction of the polymer to itself, which interacts with itself via the potential $v(\mathbf{r})$.

At first, this weak attraction would seem to be in contradiction with the screening theorem [2] that says for long chains, the statistics of individual chains are ideal. However we are considering a potential that is static only on a time scale much less than the reptation time. On that time scale, the curvilinear motion of the tube is small and we can consider what happens if we stop chain ends completely from reptating. Because of entanglements, chain motion is confined to a tube and this means that we do not expect complete screening in this case. Chain of length less than $N_{e}$ can move quite freely, but above that entanglements reduce the number of degrees of freedom. Therefore we expect that the screening of excluded volume is not complete, and the screening should scale as it would for a melt of chains of length $N_{e}$. Therefore the excluded volume parameter $\propto 1 / N_{e}[2]$.

On the scale of blobs of length of $N_{e}$, this leads to a net repulsive potential $\sim\left(1 / N_{e}\right) N_{e}^{2} / V \sim N_{e}^{-1 / 2}$, with a range of a tube diameter. This is exactly the same scale as the scale of the effective attractive potential in Eq. [6] because the height of $v$ is $\sim N_{e}^{-1 / 2}$ also with the range of a tube diameter. Therefore one expects that this weak attraction is canceled out by the excluded volume repulsion weakened by screening, in order to have ideal chains over large distances.
This argument can be turned around: the fact that we must have complete screening for arbitrarily long chains, and that screening will be imperfect for polymers confined to a tube, implies that the polymer chain must move in a weak random potential whose strength is such as to give complete cancellation of the second virial coefficient. In other words, the assumption of reptation, plus the requirement of screening, gives rise to the random potential with the strength given above.

After stressing the system, the random potential will change so that the correlation function is $v_{s}\left(\mathbf{r}-\mathbf{r}^{\prime}\right)$. The form of the potential should now be anisotropic and approximately $v_{s}=v(\Lambda \mathbf{r})$ where $\Lambda$ is the affine transformation of the unsheared coordinates to the sheared state. In the direction of maximum extension, the range of $v_{s}$ has now increased, and perpendicular to this, the range has decreased. This clearly leads to an alignment of the chains in the direction of maximum extension. To calculate the local anisotropy quantitatively is not possible without additional information. The exact form of fluctuation in Eq. 6] depends on the correlations of entanglements in these systems. This, at the moment, is unknown. Related to this, is how the imperfect screening of chains in tubes, discussed above, is modified by stretching. This is necessary to determine chain statistics in the stretched state.

The $\epsilon$ factor in Eq. 3 is $\propto N_{e}^{-1 / 4}$. This is a substantial amount of orientational bias on the scale of a tube diameter. In a melt one would also expect that chain packing would also induce further alignment locally due to local chain packing and indeed an $\epsilon=0.45$ has been reported experimentally. However in concentrated and semidilute solutions, such local packing effects would not be expected to play a large role. In this case, we expect that the mechanism discussed here would dominate.

In conclusion we have shown that induced orientational coupling of chains in a concentrated polymer solution can be understood as a consequence of the many body interactions of a polymer system undergoing reptation. Reptational motion freezes in degrees of freedom causing incomplete screening that must be balanced by a random potential causing chain attraction. This random potential has its origin in elastic fluctuations of the system [9]. When the system is stretched, the correlation in the random potential no longer screens interactions completely and causes the chain to elongate in the direction of stretch. This was analyzed directly by computer simulation using reptation dynamics and demonstrated that this orientational bias is substantial.
[1] J.D. Ferry, Viscoelastic Properties of polymers (Wiley, New York, 1980) 3rd ed.

[2] P.G. de Gennes "Scaling Concepts in Polymer Physics" Cornell University Press (1985).

[3] Michael Rubinstein and Ralph H. Colby, Polymer
Physics, Oxford University Press, 2003.

[4] P.G. de Gennes J. Chem. Phys. 55, 572 (1971).

[5] M. Doi, J. Polym. Sci., Polym. Phys. Ed. 21, 667 (1983).

[6] M. Rubinstein, Phys. Rev. Lett. 59, 1946 (1987).

[7] J.M. Deutsch, Phys. Rev. Lett. 54, 56 (1985). 
[8] J.M. Deutsch, J. Phys. France 48, 141 (1987).

[9] A.N. Semenov and M. Rubinstein, Eur. Phys. J. B 1, 87 (1998).

[10] M. Rubinstein, S.P. Obukhov, Phys. Rev. Lett. 71, 1856 (1993).

[11] C.M. Ylitalo, J.A. Kornfield, G.G. Fuller, D.S. Pearson, Macromolecules 24, 749 (1991).

[12] A.R.C. Baljon, G.G. Grest, T.A. Witten, Macromolecules 281835 (1995).

[13] F.T. Wall and F. Mandel, J. Chem. Phys. 63, 4592 (1975); I. Webman, J. L. Lebowitz, and M. H. Kalos,
J. Phys. France 41, 579 (1980).

[14] Phys. Rev. Lett. 95, 257802 (2005).

[15] W. W. Merrill, M. Tirrell, J.F. Tassin, and L. Monnerie, Macromolecules 22896 (1989).

[16] In reference 9] this quantity is calculated for an arbitrary length of chain. However the authors average the strain over the entire volume, which is correct for the case of interest here, but not in general.

[17] M. E. Cates and C. Ball, J. Phys. (France) 49, 2009 (1988). 